\title{
Verletzungen der Halswirbelsäule: ventrale Spondylodesetechniken
}

Matti Scholz, Andreas Pingel, Philipp Schleicher, Frank Kandziora

\section{Einleitung}

Als Ziele der operativen Therapie von HWS-Verletzungen sind die Dekompression komprimierter neurologischer Strukturen, die Wiederherstellung der segmentalen Stabilität und die Korrektur von ggf. vorhandenen traumatischen/posttraumatischen Fehlstellungen definiert. Folgende Prinzipien sollten bei der Wahl der adäquaten operativen Strategie beachtet werden [1,2]:

1. Es sollten nur so viele Bewegungssegmente wie unbedingt notwendig in die definitive Spondylodese miteinbezogen werden. Eine mehrsegmentale Versteifung im Bereich der Halswirbelsäule bedingt eine relevante Einschränkung der Beweglichkeit und kann sekundär die Lebensqualität des Patienten deutlich beeinträchtigen.

2. Die verletzten Bewegungssegmente bedürfen zur schnellen und endgültigen Ausheilung einer sicheren Ruhigstellung. Eine dauerhafte Versteifung eines Bewegungssegmentes ist ohnehin unumgänglich, daher sollte die chirurgische Therapie eine optimale Stabilität bei möglichst geringer Invasivität zum Ziel haben.

Unterschieden werden sollte zwischen den Begriffen Stabilisation und Spondylodese. Die Fixation von Bewegungssegmenten mit Implantaten und dem Ziel einer „temporären“ Ruhigstellung des Bewegungssegmentes sollte im allgemeinen Sprachgebrauch als segmentale Stabilisation bezeichnet werden. Von einer Spondylodese wird gesprochen, wenn zusätzlich zur Stabilisation Gelenke eröffnet und/oder Knochenmaterial angelagert wird, um eine dauerhafte knöcherne Überbrückung (Fusion) eines oder mehrerer Bewegungssegmente zu erreichen.

Seit der Einführung des ventralen Zugangs zur Halswirbelsäule durch Robinson werden weltweit traumatische Verletzungen der subaxialen Halswirbelsäule mit gutem klinischem Erfolg behandelt. Als Voraussetzung für eine definitive ventrale Spondylodese gilt bis heute eine adäquate ventrale Diskektomie, über die auch der Spinalkanal ausreichend dekomprimiert werden kann. Nach alleiniger zervikaler Diskektomie ohne anschließende intervertebrale Stabilisation/Fusion verbleibt ein instabiles Bewegungssegment. Die daraus resultierende segmentale Kyphosierung führt meist zu einer Heilung in Fehlstellung, die sich ungünstig auf die Statik der Halswirbelsäule auswirkt und die mechanische Beanspruchung der An- schlusssegmente verstärken kann. Daher wird allgemein eine intervertebrale Spondylodese unter Erhalt/Rekonstruktion der Bandscheibenraumhöhe nach Diskektomie empfohlen.

Lange Zeit wurde der autologe trikortikale Beckenkammspan als Standardtransplantat genutzt, um eine intervertebrale Fusion zu erreichen. Probleme resultierten aus der unzureichenden Primärstabilität des Beckenkammspantransplantats, sodass Hermann [3] die Verwendung einer zusätzlichen zervikalen Platte empfahl, um Komplikationen bei Verwendung des Beckenkammspans zu vermeiden. Letztendlich hat sich die Verwendung einer additiven Platte bei der ventralen Spondylodese zur Versorgung einer traumatischen HWS-Verletzung durchgesetzt [4].

Mehr und mehr verdrängt wurde der autologe Beckenkammspan durch die Einführung von metallischen Platzhaltern, die eine hohe Primärstabilität aufweisen und keine Entnahmemorbidität in der Beckenregion bedingen. Aktuell können Cages verschiedener Designs (z. B. zylindrisch, boxförmig) aus verschiedenen Materialien (z.B. Titan, PEEK) von den Herstellern bezogen werden, wobei die Verwendung einer additiven Platte weiterhin als Standard bei der Frakturversorgung gilt.

\section{Indikation zur ventralen Spondylodese}

Für Verletzungen mit neurologischen Ausfallserscheinungen verdichten sich die wissenschaftlichen Hinweise, dass eine zügige Dekompression des Spinalkanals mit nachfolgender adäquater segmentaler Stabilisation ein verbessertes neurologisches Outcome zur Folge hat [5]. Daher ist die akute traumatische Querschnittslähmung oder eine akute radikuläre Ausfallserscheinung eine absolute OP-Indikation. Ebenfalls besteht bei bildmorphologisch instabiler Fraktur eine absolute OP-Indikation, da diese zu einer neurologischen Verschlechterung des Patienten führen kann. Für die Definition einer Instabilität besteht aktuell kein allgemeingültiger Konsens, wobei im deutschsprachigen Raum A3-, A4-, B- und C-Verletzungen typischerweise als instabil angesehen werden. Radiologische Hinweise auf eine Instabilität ergeben sich gemäß der unten angegebenen Kriterien, für deren Abklärung im Zweifel funktionsradiologische Untersuchungen der Halswirbelsäule herangezogen werden können. 


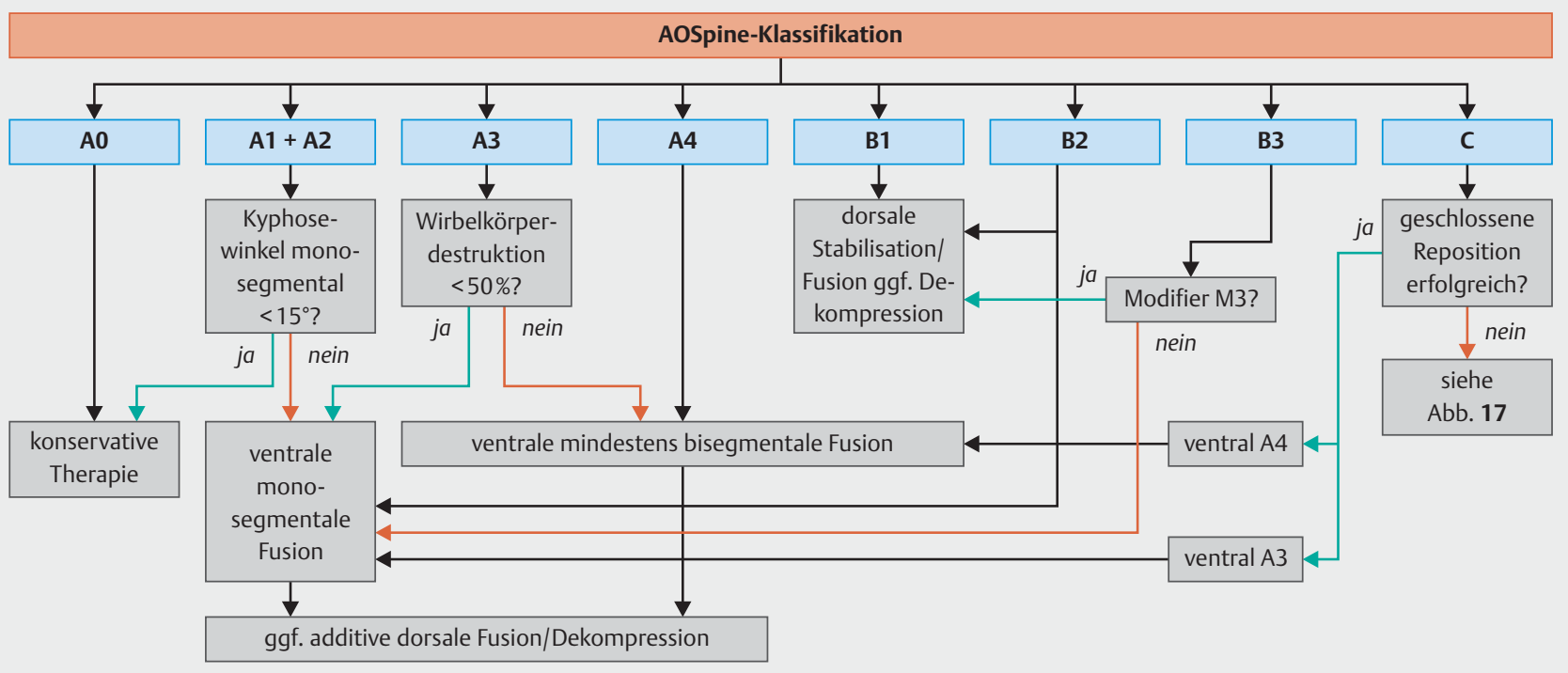

- Abb. 1 Therapieschema der Autoren für AOSpine-A- und -B-Verletzungen sowie geschlossen reponible C-Verletzungen (grüne Pfeile = ja; rote Pfeile = nein). Aus [2] mit freundlicher Genehmigung von Thieme.

Instabilitätskriterien gemäß Panjabi et al. [6]:

- anterioposteriore Translation > $3 \mathrm{~mm}$

- segmentale Kyphose $>11^{\circ}$

- Facettensubluxation > 50\% der Gelenkfläche

Prinzipiell können nahezu alle subaxialen HWS-Verletzungen primär über einen ventralen Zugang versorgt werden, wobei die weichteilschonende Präparation den größten Vorteil des ventralen Zugangs darstellt. Bei den ventralen Fusionstechniken kann zwischen einem intervertebralen - meist monosegmentalen - Fusionsverfahren („anterior cervical decompression and fusion“ ACDF) und einem mehrsegmentalen Fusionsverfahren unter Resektion eines oder mehrerer Wirbelkörper („anterior cervical corporectomy and fusion“ - ACCF) unterschieden werden.

Vor- und Nachteile des ventralen Zugangs:

Vorteile:

- einfache Lagerung (Rückenlage)

- Zugang weichteilschonend

- direkte Dekompression bei ventraler Kompression (A3-, A4-Frakturen) möglich

- kurzstreckige Versorgung möglich

- segmentale Kyphosekorrektur

- geringere Komplikationsrate (Blutverlust, Infektionsrate)
Nachteile:

- temporäre Dysphagie

- Risiko für Verletzung viszeraler Strukturen (Ösophagus und Trachea)

- Rekurrensparese oder Horner-Syndrom möglich

- offene Reposition technisch aufwendig (C-Verletzung)

Kontraindikationen für eine isolierte ventrale Fusion stellen verhakte Luxationsfrakturen (AOSpine C, F4) dar, die weder geschlossen noch offen über einen ventralen $\mathrm{Zu}$ gang reponiert werden können. Eine weitere Frakturentität, die eher primär von dorsal langstreckig stabilisiert werden sollte, stellen AOSpine-B3-Frakturen bei Morbus Bechterew (siehe Beitrag „HWS-Frakturen bei ankylosierenden Erkrankungen“ im selbigen Heft) dar.

Das nachfolgende Flussschema ( $\bullet$ Abb. 1) kann Sie - die Kenntnis der korrekten Frakturklassifikation wird vorausgesetzt - bei der Wahl der geeigneten Operationsstrategie unterstützen.

\section{Aufklärung und Operationsvorbereitung}

Bei der Aufklärung für eine ventrale Fusion sollte auf die typischen perioperativen und postoperativen Risiken des gewählten Zugangs/der Versorgungsstrategie eingegangen werden. 

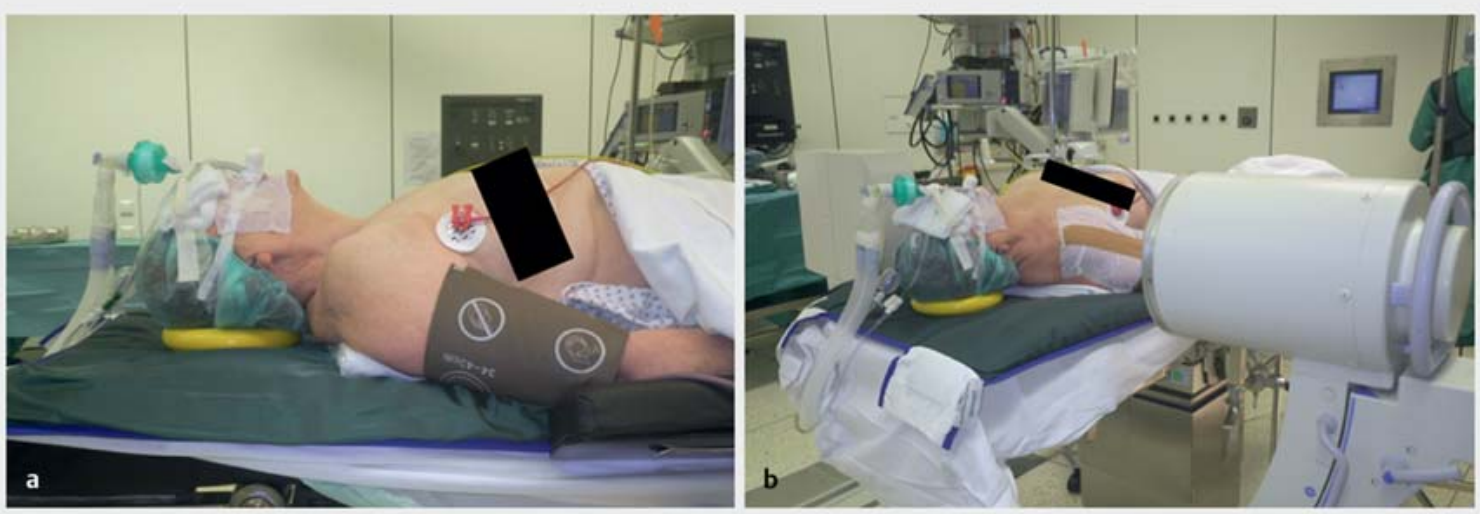

- Abb. 2 a Rückenlagerung im Kopfring mit reklinierter Halswirbelsäule. b Seitliche Durchleuchtung der HWS mit Zug an den Armen über eine Pflasterzügelung.

Zu nennende Risiken:

- Lagerungsschaden

- Verletzung von Trachea oder Ösophagus

- intraoperative Blutung (epiduraler Venenplexus, A. carotis, V. jugularis)

- Nachblutung/Hämatom mit ggf. notfallmäßiger Revisionsindikation

- Verletzung N. laryngeus recurrens $\rightarrow$ Stimmbandparese mit Heiserkeit

- Verletzung des Grenzstranges $\rightarrow$ Horner-Syndrom

- Verletzung von Nervenwurzeln/Rückenmark $\rightarrow$ Radikulopathie/Querschnittsyndrom

- Eröffnung der Dura $\rightarrow$ Durafistel

- selten Wundheilungsstörungen/Wundinfekt

- Implantatversagen/Lockerung der Implantate

- Pseudarthrose

- Anschlusssegmentproblematik

- Reststenose der Neuroforamina

Für die Operation werden die Patienten meist oral intubiert. Da der Beatmungsschlauch nach kranial über den Kopf geführt werden sollte, ist die Verwendung eines Spiraltubus zu empfehlen, um das Abknicken des Tubus mit nachfolgenden Beatmungsproblemen zu vermeiden. Zusätzlich ist eine Cuffdruckmessung notwendig, um den Cuffdruck intraoperativ regulieren zu können. Bei hochinstabiler Fraktursituation sollte ggf. videolaryngoskopisch intubiert werden, um die Halswirbelsäule so wenig wie möglich zu manipulieren. Bei Intubationsschwierigkeiten kann der ggf. anliegende Stiffneck geöffnet oder auch entfernt werden, wobei der Kopf dann manuell immobilisiert werden muss. Alternativ kann eine fiberoptische Wachintubation geplant werden. Es sollte immer eine Magensonde eingelegt werden, um den Ösophagus intraoperativ tasten zu können und dessen Lage im Zugang zu verifizieren.

\section{Merke}

OP-Tipp: Zur Reduktion des Risikos für eine Ösophagusverletzung sollte der Ösophagus durch eine Magensonde geschient werden. Dadurch kann dieser intraoperativ leichter identifiziert und geschont werden.

\section{OP Technik}

\section{Lagerung}

Für die ventrale Spondylodese wird der Patient in Rückenlage gelagert. Wichtig dabei ist eine ausreichend stabile Lagerung des Kopfes. Bei hochinstabilen Halswirbelsäulenverletzungen erbringt die Lagerung in einer MayfieldKlemme die höchste Stabilität. Alternativ kann in einer Kopfschale oder auch in einem Kopfring ( $\triangleright$ Abb. 2 a) gelagert werden. Eventuell noch anliegende Orthesen sind zu entfernen. Wenn frakturbedingt möglich, sollte die Halswirbelsäule in physiologischer Lordose eingestellt werden, um die Halswirbelsäule später nicht in kyphotischer Fehlstellung zu fusionieren. Nach erfolgter Lagerung sollte zeitnah eine Stellungskontrolle mittels eines Bildverstärkers (BV) sowohl im seitlichen als auch im a.-p. Strahlengang erfolgen, um die adäquate Lagerung und achsgerechte Stellung der Halswirbelsäule zu verifizieren. Die seitliche Durchleuchtung der unteren Halswirbelsäule kann durch die Überprojektion des Schultergürtels erschwert sein. Hier können die Arme ggf. vorsichtig mittels Zugeinrichtung oder Pflasterzügelung nach kaudal gezogen werden, um eine ausreichende intraoperative Visualisierung der subaxialen Halswirbelsäule im seitlichen Strahlengang zu ermöglichen ( $\bullet$ Abb. 2 b).

\section{Zugang}

Der anterolaterale (ventrale) Zugang ist der Standardzugang zur Versorgung von Halswirbelsäulenverletzungen. Dieser Zugang erfolgt weichteilschonend streng 


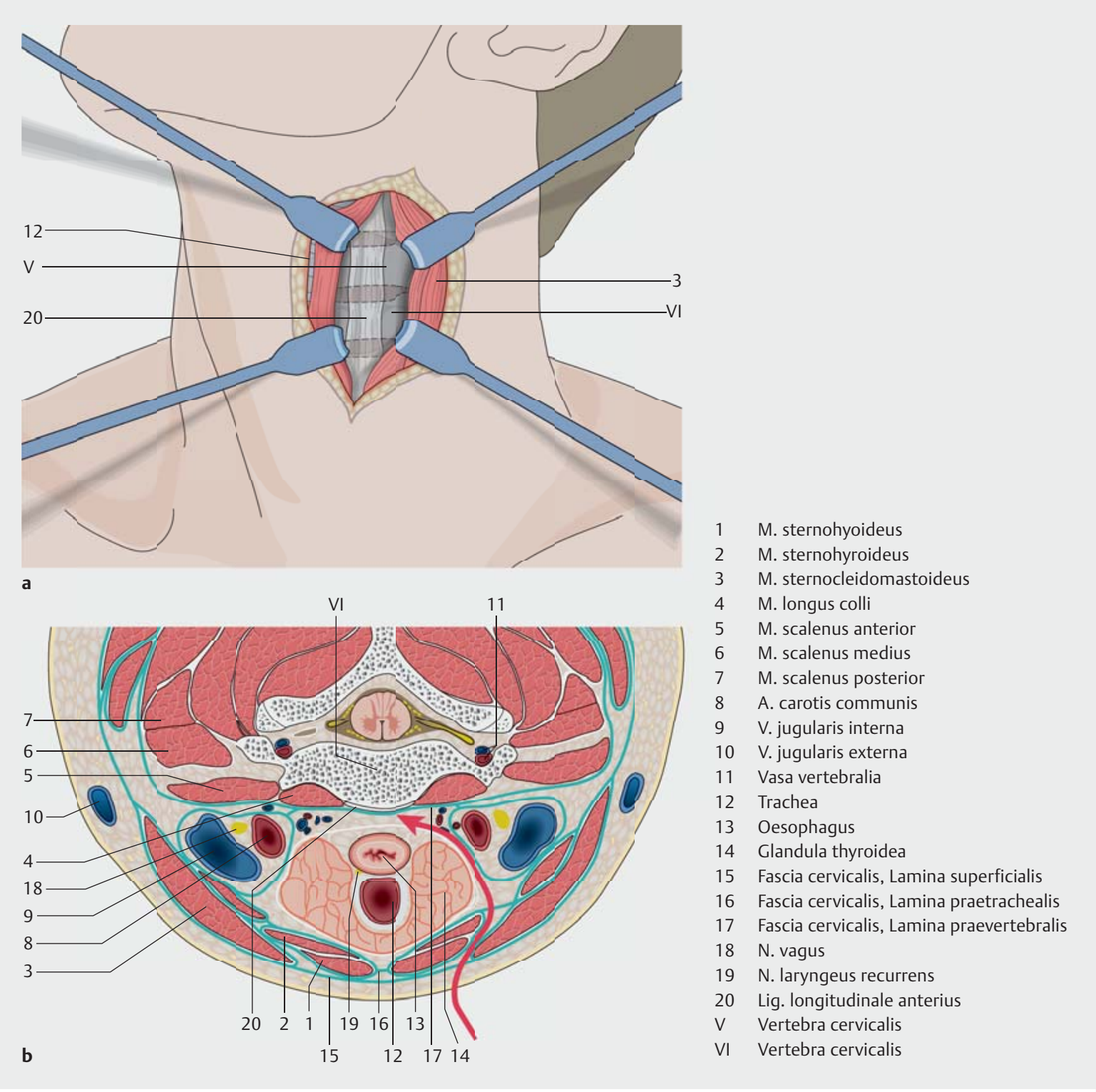

- Abb. 3 Darstellung der Wirbelkörpervorderfläche per linksseitigem Smith-Robinson-Zugang. Aus [2] mit freundlicher Genehmigung von Thieme.

medial des M. sternocleidomastoideus, entlang anatomisch vorgegebener Verschiebeschichten ( $\bullet$ Abb. 3). Der Hautschnitt kann horizontal ( $\bullet$ Abb. 4) ausgehend von der Mittellinie bis zum M. sternocleidomastoideus in Projektion auf das destruierte Bewegungssegment (Cloward-Zugang) oder auch longitudinal direkt am Vorderrand des M. sternocleidomastoideus erfolgen (SmithRobinson-Zugang). Der Cloward-Zugang eignet sich bei monosegmentalen und bisegmentalen Pathologien und ergibt das kosmetisch günstigere Ergebnis. Bei bi- oder vor allem bei multisegmentalen Pathologien sollte der Smith-Robinson-Zugang gewählt werden, da sich dieser unproblematisch nach kranial oder kaudal erweitern lässt.
Ob der Zugang von links oder rechts durchgeführt wird, obliegt der klinischen Erfahrung und Präferenz des Chirurgen. Nach erfolgtem Hautschnitt, subtiler Blutstillung und Eröffnung des Platysmas sollten die weitere Präparation und Dissektion stumpf erfolgen, um das Risiko für die Verletzung vaskulärer, viszeraler und neuraler Strukturen zu reduzieren. Dabei ist es hilfreich, nach Identifikation des M. sternocleidomastoideus ( $\bullet \mathbf{A b b} \mathbf{5} \mathbf{5} \mathbf{a})$ die Trachea und den Ösophagus mit einem Haken (z. B. ZenkerHaken) nach medial zu mobilisieren und die A. carotis zu tasten. Diese Arterie muss dabei zwingend lateral des Präparationsfeldes getastet und belassen werden.

Bei korrekter Präparation kann dann in der Tiefe der ventrale Anteil der Halswirbelsäule getastet werden 


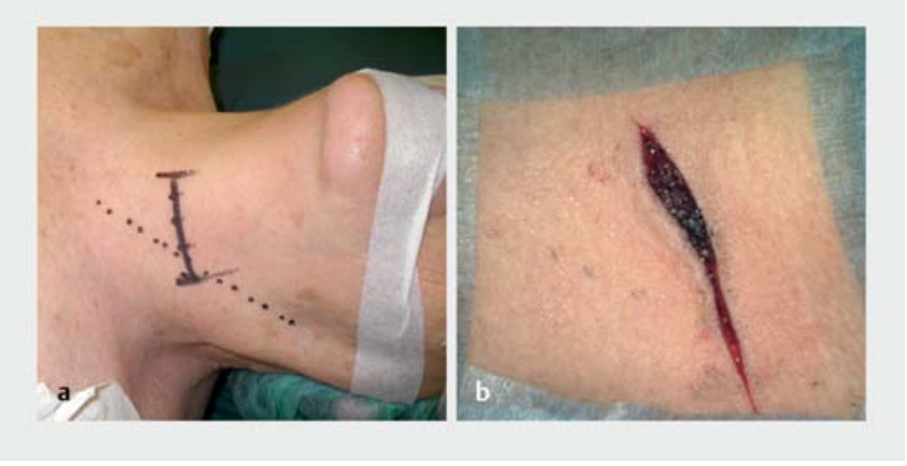

- Abb. 4 a Planung des horizontalen linksseitigen Cloward-Zugangs medial des M. sternocleidomastoideus. b Situs nach Hautschnitt.

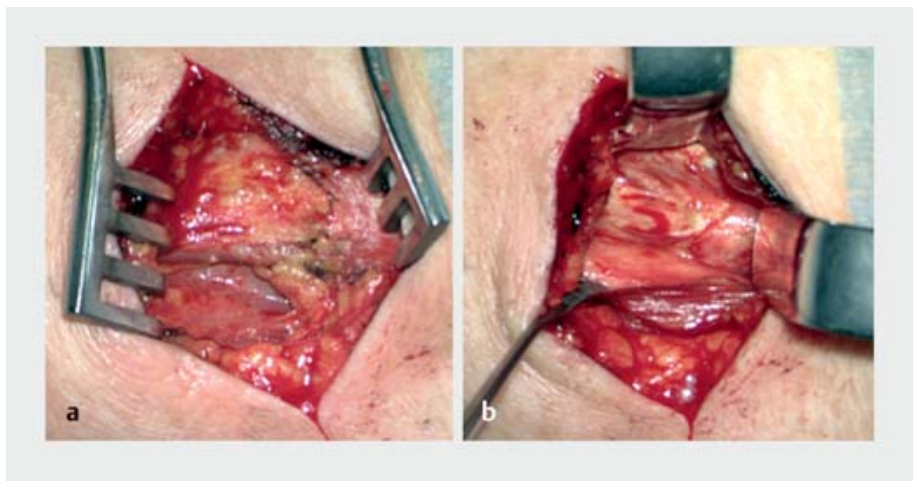

- Abb. 5 a Situs nach Eröffnung des Platysmas mit Darstellung des M. sternocleidomastoideus. b Situs nach stumpfer Präparation und Darstellung des ventralen Anteils der Halswirbelsäule.
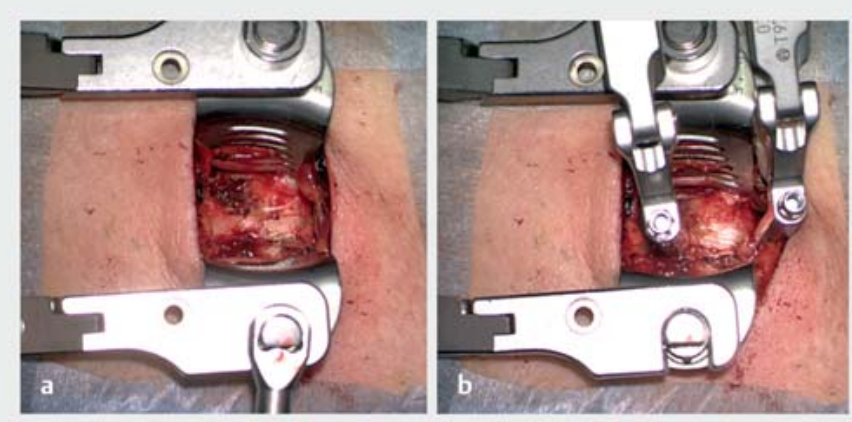

- Abb. 6 a Situs nach Einbringen des Weichteilsperrers unterhalb des M. longus colli. b Situs nach zusätzlichem Einbringen des Caspar-Sperrers.
( $\vee$ Abb. 5b). Gegebenenfalls muss die darüber liegende tiefe Halsfaszie mit der Schere eröffnet werden. Bei der Exposition empfiehlt es sich, das kranial und kaudal der Läsion gelegene Segment ebenso zu exponieren, um die Retraktionskräfte zu reduzieren und das spätere Anbringen der Platte zu erleichtern. Danach sollte der ventral auf der Halswirbelsäule liegende M. longus colli beidseits vorsichtig von medial mobilisiert werden, um den Weich-

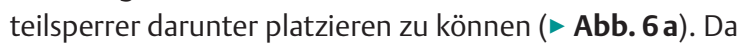
auf dem Muskel sympathische Nervenfasern verlaufen, sollte die Verwendung von monopolarem Strom oder eine ausgedehnte bipolare Koagulation vermieden werden. Eine Läsion dieses sympathischen Nervengeflechtes kann ein temporäres oder ggf. dauerhaftes Horner-Syndrom zur Folge haben.

\section{Merke}

OP-Tipp: Die Anpassung und Reduktion des Cuffdruckes nach dem Einbringen der Weichteilsperrer kann die Häufigkeit einer Rekurrensparese reduzieren [7].

Bei korrektem Zugang sollte abschließend das GefäßNerven-Bündel (A. carotis, V. jugularis, N. vagus) lateral des Zugangs gelegen sein und sich die Trachea wie auch der durch eine Magensonde geschiente Ösophagus medial des Zugangs tasten lassen.

\section{Cave}

Cave: Eine Präparation lateral der A. carotis und spätere Retraktion nach medial erhöht das Risiko für eine Gefäß-/Nerven-Verletzung und kann eine Minderdurchblutung des Gehirns bzw. einen Schlaganfall bedingen.

Durch Einbringen jeweils eines Steinmann-Pins kranial und kaudal des verletzten Segmentes (ACDF) oder kranial und kaudal des verletzten Wirbelkörpers (ACCF) und Verwendung eines Distraktors (z. B. Caspar-Distraktor) kann die spätere segmentale Dekompression vereinfachen. Die Caspar-Pins sollten streng in der Mittellinie eingebracht werden, damit das Bohrloch nicht mit den späteren Schraubenkanälen der Platte konkurriert. In den meisten Fällen ist eine parallele Ausrichtung der Pins zum Bandscheibenraum zu empfehlen, um ein paralleles Aufspreizen des Bandscheibenraumes zu ermöglichen ( A Abb. 6b).

\section{Cave}

Cave: Eine segmentale Überdistraktion - insbesondere bei diskoligamentärer Instabilität - sollte vermieden werden!

\section{Dekompression}

Die ventrale Dekompression lässt sich am besten unter Verwendung eines Mikroskops oder alternativ unter Verwendung einer Lupenbrille durchführen. Die Autoren präferieren die Verwendung des Mikroskops wegen der 
hervorragenden Ausleuchtung des Operationsfeldes und der entsprechenden optischen Vergrößerung. Im Weiteren empfiehlt sich die Verwendung des Mikroskops auch unter Ausbildungskriterien, da Operateur und Assistent das Gleiche sehen. Im Gegensatz dazu hat bei Verwendung einer Lupenbrille nur der Operateur einen adäquaten Einblick in den Operationssitus.

Bei geplanter ACDF ist zur ventralen Dekompression des Myelons häufig eine Diskektomie ausreichend (A3-Fraktur, B2- und C-Verletzung mit ventraler A0-, A1- oder A3-Komponente). Liegt eine A4-Fraktur oder eine B2-/CVerletzung mit ventraler A2- und/oder A4-Komponente vor, ist meist eine Korporektomie notwendig, die ggf. auch über mehrere Wirbelkörper/Segmente erfolgen kann.

\section{ACDF}

Falls die Bandscheibe nicht schon durch die Verletzung zerrissen ist, wird diese mit dem 11er-Skalpell zunächst kaudal und dann kranial eröffnet ( $\bullet \mathbf{A b b} \mathbf{7} \mathbf{a}$ ). Dabei sollte von der Mittelinie bis zum linken und rechten Processus uncinatus geschnitten werden, wobei der Processus uncinatus eine Eröffnung des lateralen Anulus bzw. eine Läsion der Vertebralarterie verhindert. Es sollte nicht tiefer als $10 \mathrm{~mm}$ inzidiert werden, um eine akzidentelle Eröffnung der Dura zu verhindern. Anschließend wird die Bandscheibe mittels Fasszange, scharfem Löffel und Kürette entfernt. Dabei sollte auch der knorpelige Überzug der Endplatten entfernt werden, bis petechiale Blutungen auftreten. Anschließend muss noch die Hinterkante des frakturierten Wirbelkörpers unterschnitten werden, falls eine relevante spinale Stenose besteht. Ob eine Eröffnung des hinteren Längsbandes notwendig ist, hängt vom Ausmaß der Spinalkanalstenose, ggf. intraspinal liegenden Bandscheibensequestern oder vom Vorhandensein eines intraspinalen Hämatoms ab. Können diese Pathologien z.B. im MRT sicher ausgeschlossen werden, muss das hintere Längsband nicht zwingend eröffnet werden. Im Zweifelsfall ist eine Eröffnung des hinteren Längsbandes und die chirurgische SpinalkanalClearance zu empfehlen ( $\bullet$ Abb. 7 b). Bei Radikulopathie aufgrund einer frakturbedingten Enge des Neuroforamens ist zusätzlich eine Unkoforaminotomie notwendig, um den Spinalnerv von ventral zu entlasten.

\section{Cave \\ Cave: Bei der Unkoforaminotomie sollte beachtet werden, dass die A. vertebralis im Foramen transver- sarium durch knöcherne Fragmente verletzt sein kann, aber auch iatrogen eine Verletzung möglich ist.}

Bei möglicherweise auftretenden venösen epiduralen Blutungen sollte eine sichere und schnelle Blutstillung oberste Priorität haben. Hier kann eine epidurale Instillation von Wasser, die vorsichtige bipolare Koagulation oder temporäre Einlage von Hämostyptika mit Hirnwatte

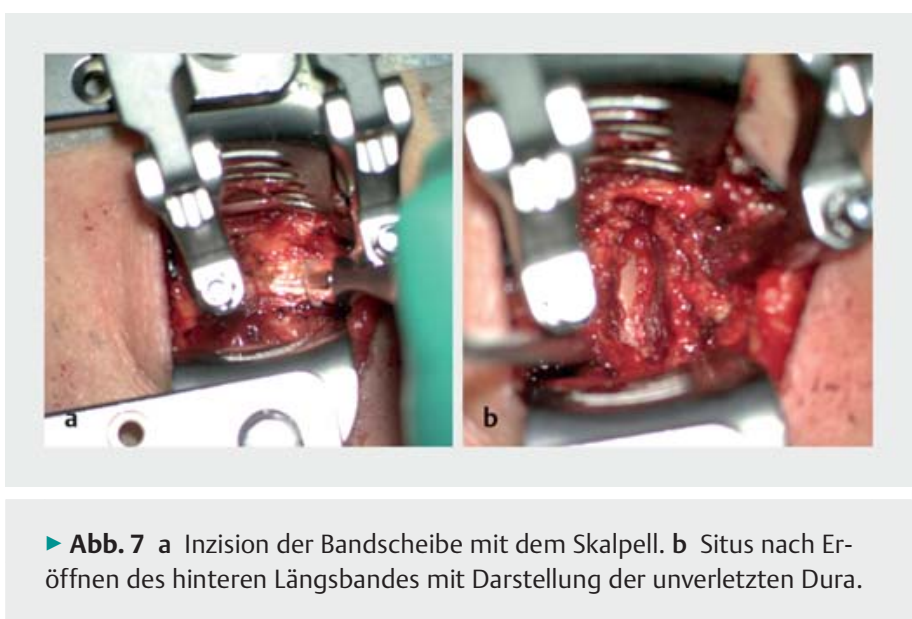

zu einer Blutstillung führen. Bei persistierender venöser Blutung kann ein injizierbares Hämostyptikum (z. B. FloSeal ${ }^{\circledR}$ ) verwendet werden, um eine schnelle und sichere Blutstillung zu erreichen. Abschließend sollte eine vollständige Dekompression des Spinalkanals und des Neuroforamens mit dem Tasthaken unter seitlicher Durchleuchtung dokumentiert werden.

\section{ACCF}

In Analogie zur Dekompression bei der ACDF erfolgt bei geplanter Korporektomie zunächst die Entfernung der kranialen und kaudalen Bandscheibe inklusive Dekompression der Hinterkante, falls möglich. Danach kann der zerstörte Halswirbel mittels Luer, Stanze oder Fräse bis auf die Hinterkante abgetragen werden. Die Hinterkante kann dann entweder mit der Stanze oder der Diamantfräse entfernt werden, wonach der Spinalkanal ausreichend dekomprimiert sein sollte. Fakultativ kann dann noch eine Foraminotomie durchgeführt werden, sollte eine relevante knöcherne Foramenstenose bestehen. Die Seitenwände des frakturierten Wirbelkörpers können i.d.R. in situ verbleiben. Eventuell bestehende venöse Blutungen aus der Restspongiosa können gut mit der Diamantfräse gestoppt werden.

\footnotetext{
Cave

Cave: Bei eventuell geplanter Resektion der Seitenwände des frakturierten Wirbelkörpers sollte die anatomische Nähe der Vertebralarterie bedacht werden, die direkt neben der Seitenwand des Wirbelkörpers verläuft ( $>$ Abb. 8 ).
}

\section{Implantate}

Die Spondylodese kann entweder intersegmental (mono-, bi- oder auch multisegmental) mittels Cage und additiver ventraler Platte erfolgen ( $\triangleright$ Abb. 9 und 10). Wichtig dabei ist die korrekte Einstellung der segmentalen Lordose. Dafür sollten entsprechend lordotisch geformte Cages gewählt und die ventrale Platte zusätzlich lordotisch vor- 


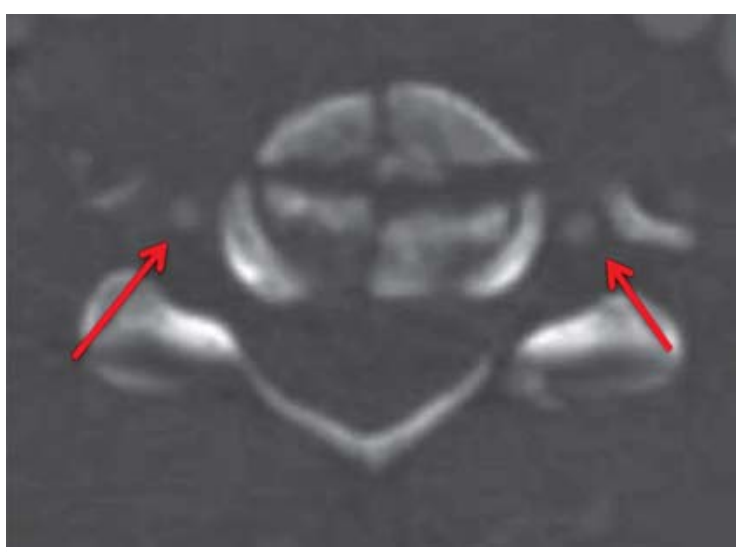

- Abb. 8 Axialer CT-Schnitt mit Kontrastmitteldarstellung der $A$. vertebralis und Demonstration der Nähe der Vertebralarterie zur Wirbelkörperseitenwand.

gebogen werden. Bei ventraler kompletter Berstung (AOS A4) oder mehrsegmentaler Operation mit Notwendigkeit zur Korporektomie können metallische Wirbelkörperersatzimplantate (z. B. expandierbare Cages) mit zusätzlicher ventraler Platte verwendet werden ( $\triangleright$ Abb. 11). Alternativ kann anstatt metallischer Cages auch ein autologes trikortikales Beckenkammspantransplantat verwandt werden. Aufgrund der Entnahmemorbidität und der unzureichenden mechanischen Primärstabilität wird zunehmend auf die Verwendung von autologen Knochenspänen verzichtet.

Nach Beendigung der Dekompression werden dann Probe-Cages unter seitlicher Röntgenkontrolle vorsichtig in den Diskektomie-/Korporektomiedefekt eingebracht, um die adäquate Implantatgröße zu bestimmen. Eine Überdistraktion durch einen zu hohen Cage sollte hierbei vermieden werden. Nach Auswahl des geeigneten Implantats und wird dieses auf dem Cage-Halter montiert. Die Cages können mit dem bei der Dekompression gewonnenen autologen Knochenmaterial gefüllt werden bzw. dieses kann in der Korporektomiesituation lateral der Cages für die ventrale Spondylodese angelagert werden. Ist nicht genügend autologer Knochen vorhanden, ergibt die Füllung der Cages mit z.B. mit $\beta$-Trikalziumphosphat ebenso hervorragende Fusionsergebnisse [8].

Anschließend wird der Cage unter seitlicher Röntgenkontrolle bis zum gewünschten Sitz in den Diskektomie-/Korporektomiedefekt eingebracht. Wirbelkörperersatzimplantate werden ggf. expandiert, um den Defekt adäquat zu überbrücken. Auch hier sollte wiederum eine Überdistraktion vermieden werden. Anschließend wird der CageHalter entfernt und ebenso der Caspar-Distraktor demontiert. Anschließend sollte die korrekte Implantatlage auch im a.-p. Strahlengang dokumentiert werden.

\section{Cave}

Cave: Ein zu tiefes Einbringen (Überschreitung der Hinterkante) sollte unbedingt vermieden werden, um eine Kompression des Duralsackes zu vermeiden. OP-Tipp: Venöse Blutungen aus den Bohrlöchern für die Caspar-Pins können mit Knochenwachs zügig gestoppt werden.

Nach dem Einbringen des Cages sollte eine zusätzliche ventrale Platte aufgebracht werden, um das Bewegungssegment/die Bewegungssegmente zu stabilisieren. Diese wird entweder mit mono- oder bikortikalen Schrauben sicher im Knochen verankert. Bei Verwendung einer winkelstabilen Platte-Schrauben-Verbindung hat sich die monokortikale Schraubenplatzierung bewährt $[9,10]$.

Wichtig ist die Auswahl einer geeigneten Länge der Platte, die nicht mit dem angrenzenden Bandscheibenraum interferieren sollte. Empfohlen wird hier, einen Abstand von ca. 2-3 mm bis zum angrenzenden Bandscheibenraum einzuhalten. Um auch in der Frontalebene eine adäquate Ausrichtung der Platte sicherzustellen, wird die temporäre Plattenfixierung mit mindestens 2 Spikes und anschließender a.-p. BV-Kontrolle vor definitiver Bohrung und Verschraubung der Platte empfohlen. Die Bohrung sollte dann mit entsprechenden Bohrern mit Tiefenanschlag unter seitlicher BV-Kontrolle erfolgen, um eine adäquate Trajektorie der Bohrung sicherzustellen.

Dann wird der Situs gespült und der Weichteilsperrer vorsichtig entfernt. Abschließend erfolgen die Überprüfung und Dokumentation der korrekten Lage der Instrumentation und des korrekten Alignments der Halswirbelsäule in 2 Ebenen mittels BV.

\section{Wundverschluss}

Die Autoren empfehlen standardmäßig eine prävertebrale Redon-Drainage einzulegen, die ggf. an der Haut angenäht werden sollte. In Abhängigkeit der Blutungssituation kann diese entweder als Sog- oder als Ablaufdrainage verwendet werden. Die Wunde wird mehrschichtig verschlossen, wobei für das Platysma und die Subkutis ein resorbierbares Nahtmaterial verwandt wird. Der Hautverschluss erfolgt dann entweder mittels Klebung (z.B. Dermabond ${ }^{\circledR}$ ) oder Naht, wobei die intrakutane Naht ein kosmetisch besseres Ergebnis im Vergleich zur Donati-Rückstichnaht ergibt.

\section{Ventrale Fusion bei verhakter Facetten- gelenksluxation (AOSpine C, F4)}

Frakturen mit Translation werden als C-Verletzungen klassifiziert. Um die Fusion in achsgerechter Stellung zu ermöglichen, ist die adäquate Reposition zwingend erforderlich. Vor Reposition ist die Durchführung einer MRTUntersuchung zu empfehlen, um nach einem Bandschei- 


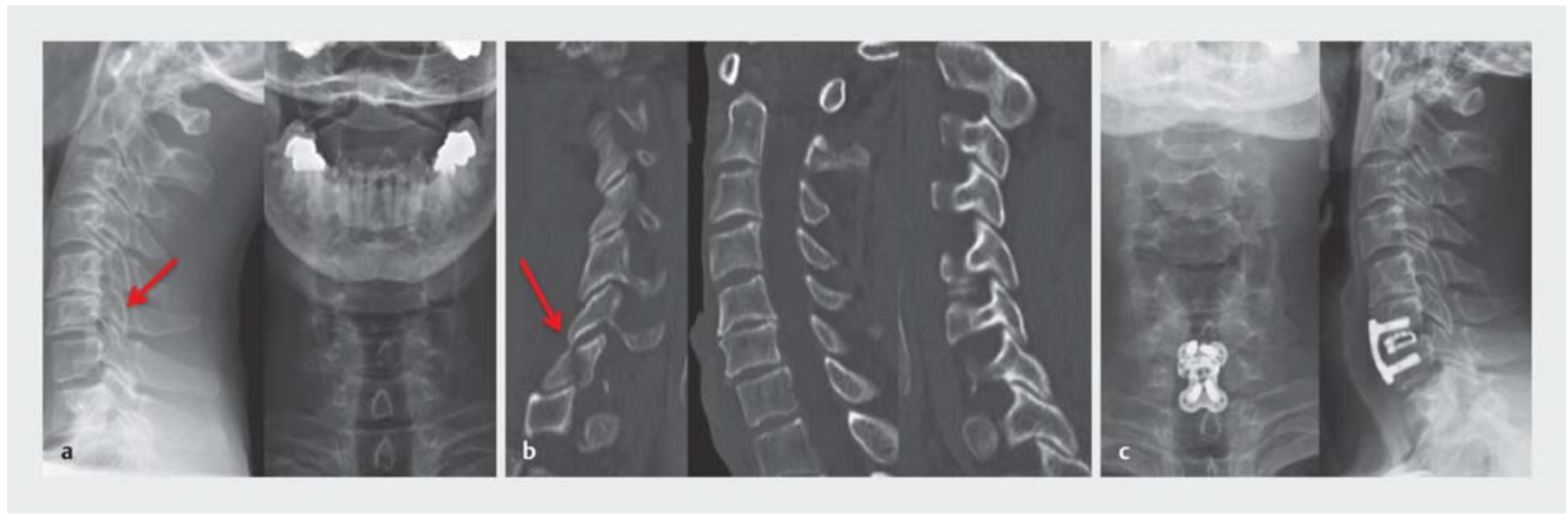

- Abb. 9 42-jähriger neurologisch unauffälliger Patient nach Sportunfall. a Nativradiologische Darstellung einer Spondylolisthese HWK VI/VII. b CT-Darstellung der unilateralen Facettengelenksfraktur HWK VI/VII. c Nativradiologische Verlaufskontrolle 6 Monate nach Reposition und ventraler Spondylodese per ACDF.

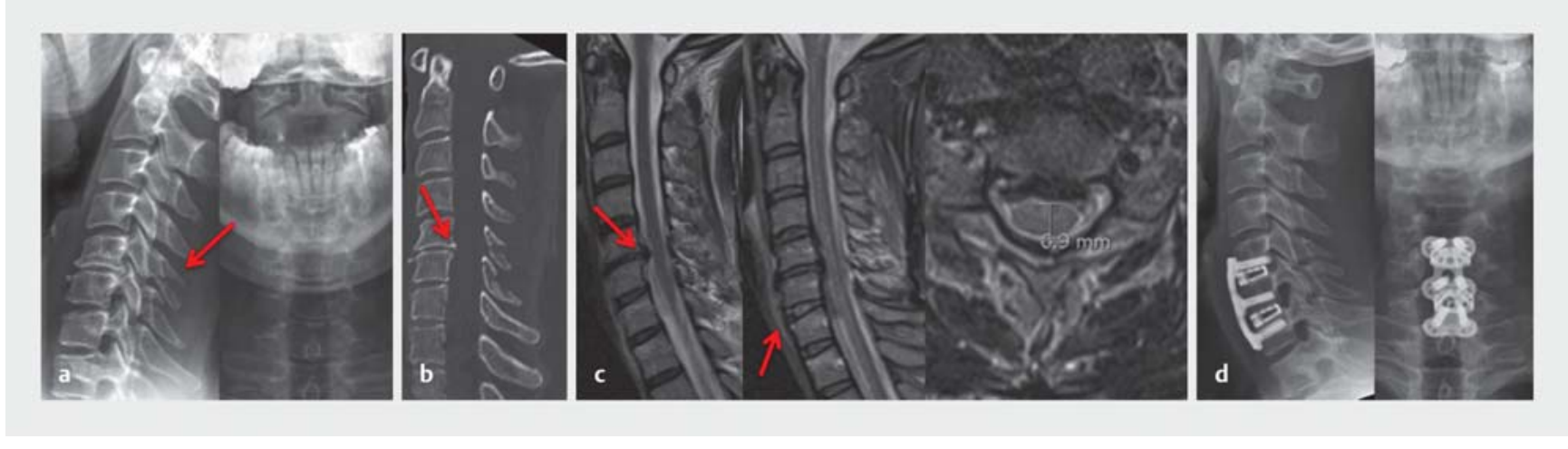

- Abb. 10 35-jährige Patientin mit Contusio spinalis nach Fahrradsturz. a Nativradiologische Darstellung der Dornfortsatz-/Laminafraktur HWK V + VI. b Sagittale CT-Rekonstruktion, roter Pfeil: Spondylchondrose mit Retrospondylophyt. c MRT mit Darstellung einer spinalen Stenose sowie Darstellung der Verletzung des vorderen Längsbandes HWK VI/VII. d Röntgenverlaufskontrolle 12 Monate nach bisegmentaler ventraler Fusion (ACDF).

bensequester zu fahnden. Dieser könnte im Rahmen der Reposition zu einer Myelonkompression führen und eine neurologische Verschlechterung bedingen. Unterschieden werden muss zwischen geschlossen reponierbaren und geschlossen nicht reponierbaren Verletzungen ( Abb. 12). Nach erfolgreicher geschlossener Reposition ist die standardisierte ventrale Dekompression und Fusion möglich, die in Abhängigkeit von der ventralen Destruktion per ACDF (ventral A3-Fraktur) oder per ACCF (ventral A4-Fraktur) erfolgen kann. Durch die ventrale Dekompression kann ein potenzieller intraspinaler Bandscheibensequester in den meisten Fällen unkompliziert entfernt werden. Häufig ist aber aufgrund der ausgeprägten dorsalen ligamentären Instabilität noch eine additive dorsale Zuggurtung notwendig. Diese kann einzeitig oder zweizeitig durchgeführt werden.
Bei geschlossen nicht reponierbarer Fraktur muss eine offene Reposition durchgeführt werden ( $\bullet$ Abb.13a, b). Diese kann, je nach Erfahrung des Operateurs, von ventral oder von dorsal durchgeführt werden.

Die größte Sicherheit für den Patienten ergibt sich bei primär ventralem Zugang und zunächst Durchführung der Diskektomie und Spinalkanal-Clearance. Daran sollte sich der Versuch der ventralen Reposition anschließen, wobei die Kompression divergierend eingebrachter Caspar-Pins ( $\bullet$ Abb. 13c) und die Flexion der HWS unter moderatem Längszug hilfreich sind. Ist die offene ventrale Reposition erfolgreich, kann primär zunächst nur von ventral mittels Cage und Platte fusioniert werden. In Abhängigkeit von Frakturausmaß, Distension der Facettengelenke und Ausmaß der residualen Instabilität kann eine additive dorsale Zuggurtung ein- oder zweizeitig durchgeführt werden ( $\bullet$ Abb. 13d-f). Ist die ventrale Reposi- 

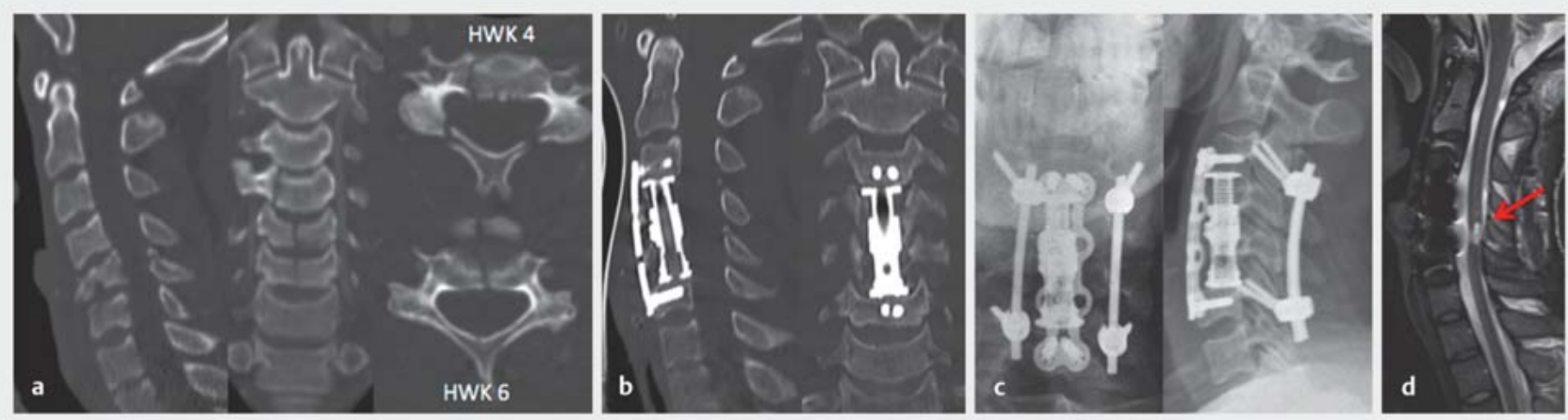

- Abb. 11 19-jähriger Patient nach Motorradunfall mit komplettem Querschnitt ASIA A bei HWS-Mehretagenverletzung. a Frakturmorphologie im CT. b Zustand nach primärer Korpororektomie und WK-Ersatz HWK IV+V. c Zustand nach dorsaler additiver Fusion HWK III-VI. d MRT nach 12 Monaten mit Darstellung des frakturbedingten Myelonschadens.

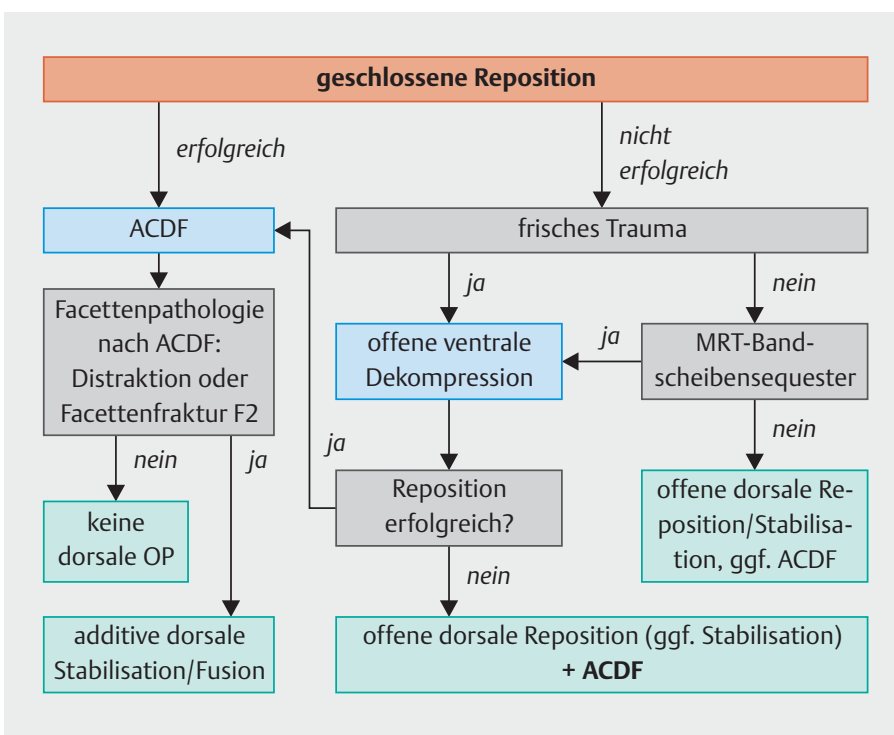

- Abb. 12 Behandlungsschema der Autoren bei verhakten Luxationsfrakturen (AOS C). Aus [2] mit freundlicher Genehmigung von Thieme.

tion nicht erfolgreich, ist nach Diskektomie die dorsale Reposition und ggf. Stabilisation durchzuführen. Danach sollte erneut von ventral zugegangen werden, um den Spinalkanal auszutasten und die Stabilisation/Fusion mittels Cage und additiver Platte zu vollenden. Das folgende Fließschema wird vonseiten der Autoren bei verhakten Luxationen verwandt.

\section{Nachbehandlung}

In Abhängigkeit von der Frakturmorphologie und vom Ausmaß der Instabilität der dorsalen Säule ist die Indikation zur additiven dorsalen Zuggurtung zu evaluieren. Als mögliche Indikation für eine additive dorsale Fusion nach ACDF/ACCF gelten [1, 2]:

- Hyperflexionsverletzung mit segmentaler Restkyphose

- Überdistraktion der Facettengelenke

- inadäquate Knochenqualität

- mehrsegmentale Korporektomie und WK-Ersatz

- primär ventrale Versorgung von Verletzungen bei ankylosierender Erkrankung der HWS

Bei geplanter zweizeitiger Versorgung sollte in diesen Fällen ggf. überbrückend eine Zervikalorthese verordnet werden. In allen anderen Fällen wird mit der operativen ventralen Versorgung eine übungsstabile Situation angestrebt. Eine Zervikalorthese ist bei stabiler Versorgung daher nicht erforderlich. Die Mobilisation des Patienten beginnt, soweit bei potenziellen Begleitverletzungen möglich, am 1. postoperativen Tag. Eine tägliche Wundkontrolle ist erforderlich.

Cave

Cave: Sollte eine Sogdrainage verwandt worden sein, sollte vor Entfernung der Sog neutralisiert werden, um vaskuläre Komplikationen (Nachblutung) zu vermeiden.

Während der ersten 6 Wochen sollte zur Unterstützung der knöchernen Fusion jegliche die Halswirbelsäule mobilisierende Krankengymnastik unterbleiben. Eine Röntgenkontrolle ist spätestens nach 12 Wochen zu empfehlen, um eine Implantatkomplikation auszuschließen. Sollte nativradiologisch der Verdacht auf eine Implantatkomplikation aufkommen, ist eine computertomografische Diagnostik (CT) zu empfehlen. Routinemäßige CT-Verlaufskontrollen zur Dokumentation des Fusionsstatus werden aus Gründen des Strahlenschutzes nicht empfohlen. Nach spätestens 12 Wochen kann der Patient dann 


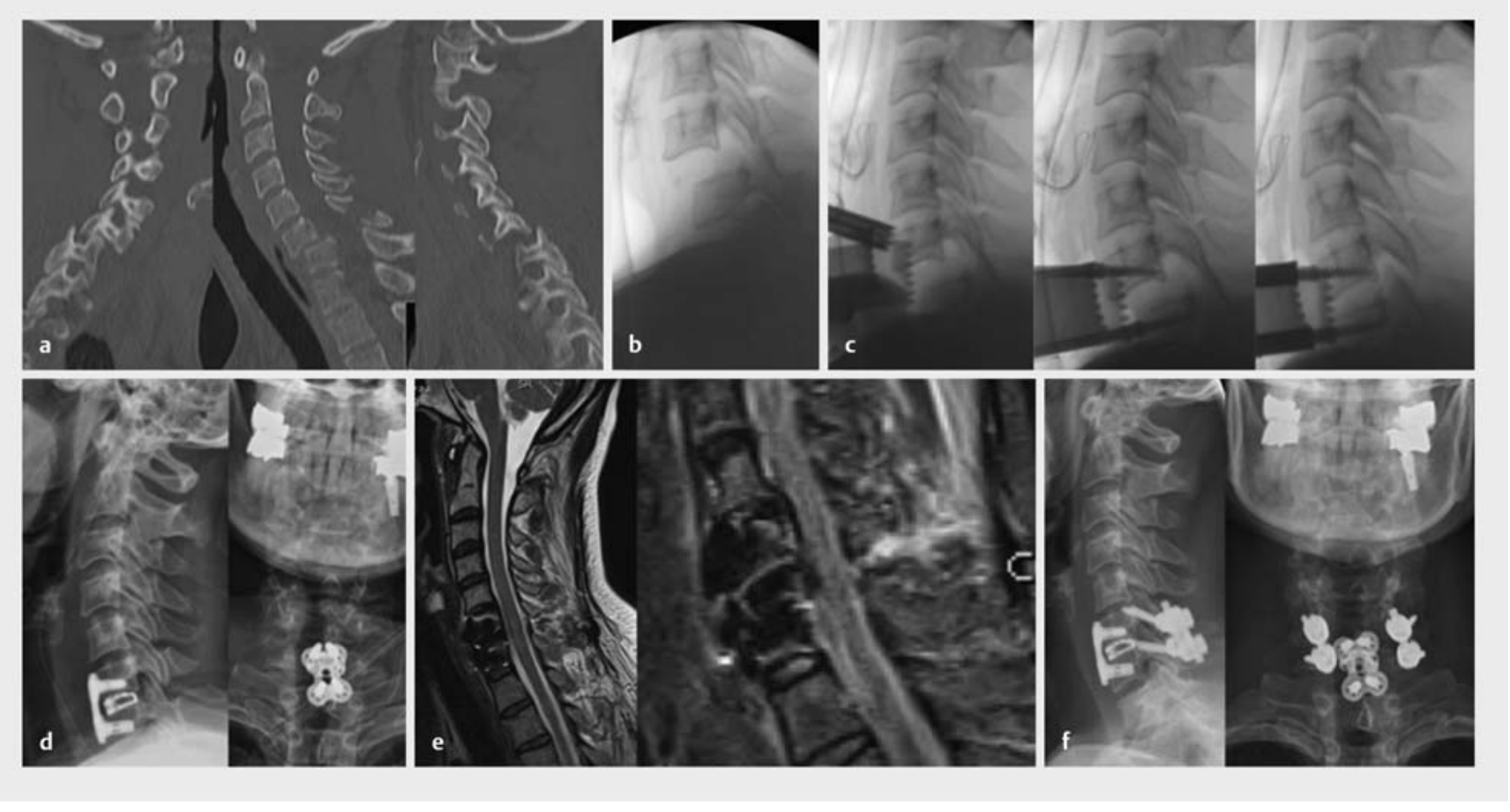

- Abb. 13 38-jährige Patientin nach Pferdesturz mit inkomplettem Querschnitt ASIA B bei HWK-VI/VII-Luxation und nebenbefundlicher idiopathischer Skoliose. a Sagittale CT-Rekonstruktion mit bilateral luxierten und verhakten Facetten. b Gescheiterter Versuch der geschlossenen Reposition. c Verlauf der erfolgreichen offenen Reposition. d Röntgen der HWS nach ACDF. e MRT postoperativ mit Darstellung der insuffizienten dorsalen Zuggurtung. $f$ HWS-Röntgenbild nach additiver dorsaler Zuggurtung (7 Monate Follow-up) bei komplett rückläufiger Neurologie (ASIA E). Aus [2] mit freundlicher Genehmigung von Thieme.

die Halswirbelsäule zunehmend belasten, und es kann eine aktive Krankengymnastik verordnet werden.

\section{Komplikationen}

Bei Komplikationen nach ventraler Spondylodese an der HWS kann zwischen intraoperativen und postoperativen Komplikationen unterschieden werden.

\section{Intraoperative Komplikationen}

\section{Parese des N. laryngeus recurrens}

Dieser Nerv ist während der Präparation beim Zugang und durch den intraoperativen Druck des Retraktors gefährdet. Patienten mit einer Läsion des N. laryngeus recurrens klagen postoperativ über Heiserkeit, und es stellt sich ein einseitiger Stimmbandstillstand bei der Laryngoskopie dar. Die Häufigkeit von temporären Beschwerden wird in der Literatur mit $11 \%$ und von permanenten Beschwerden mit 2-8\% angegeben [7,11]. Ob die Wahl der Zugangsseite die Rate der Nervenläsionen reduzieren kann, wird seit Langem kontrovers diskutiert. Der Zugang von rechts ist aber zumindest theoretisch mit einer höheren Rate von Läsionen des N. laryngeus recurrens vergesellschaftet. Andererseits können Druckläsionen bei zu hohem Cuffdruck ebenfalls zu einer Störung des N. laryngeus recurrens führen. Daher sollte nach dem
Platzieren der Weichteilsperrer der Tubuscuff kurzzeitig entblockt und dann mit maximal $20-25 \mathrm{mmHg}$ geblockt werden, um eine optimale Lage des Tubuscuffs und eine günstigere Druckverteilung zu erreichen. Die Rate von postoperativen Rekurrensparesen kann dadurch verringert werden [7].

\section{Horner-Syndrom}

Eine intraoperative Läsion des sympathischen Grenzstranges kann zu dem selten postoperativ auftretenden Symptomkomplex von Miosis, Ptosis und Enophthalmus (Horner-Syndrom) führen $[12,13]$. Ursächlich ist meist eine zu ausgedehnte mono- oder bipolare Koagulation auf dem M. longus colli, auf dessen lateralem Rand das sympathische Nervengeflecht verläuft. Zur Prophylaxe sollte nicht mit monopolarem Strom im Bereich des M. longus colli gearbeitet werden, die bipolare Blutstillung sollte sparsam erfolgen und der Muskel nur schonend, ausgehend vom medialen Rand, nach lateral mobilisiert werden.

\section{Querschnittlähmung}

Sowohl die Lagerung des Patienten als auch potenzielle Repositionsmanöver sollten nur sehr vorsichtig und unter regelmäßigen BV-Kontrollen durchgeführt werden, um eine potenzielle Kompression des Rückenmarks zu ver- 

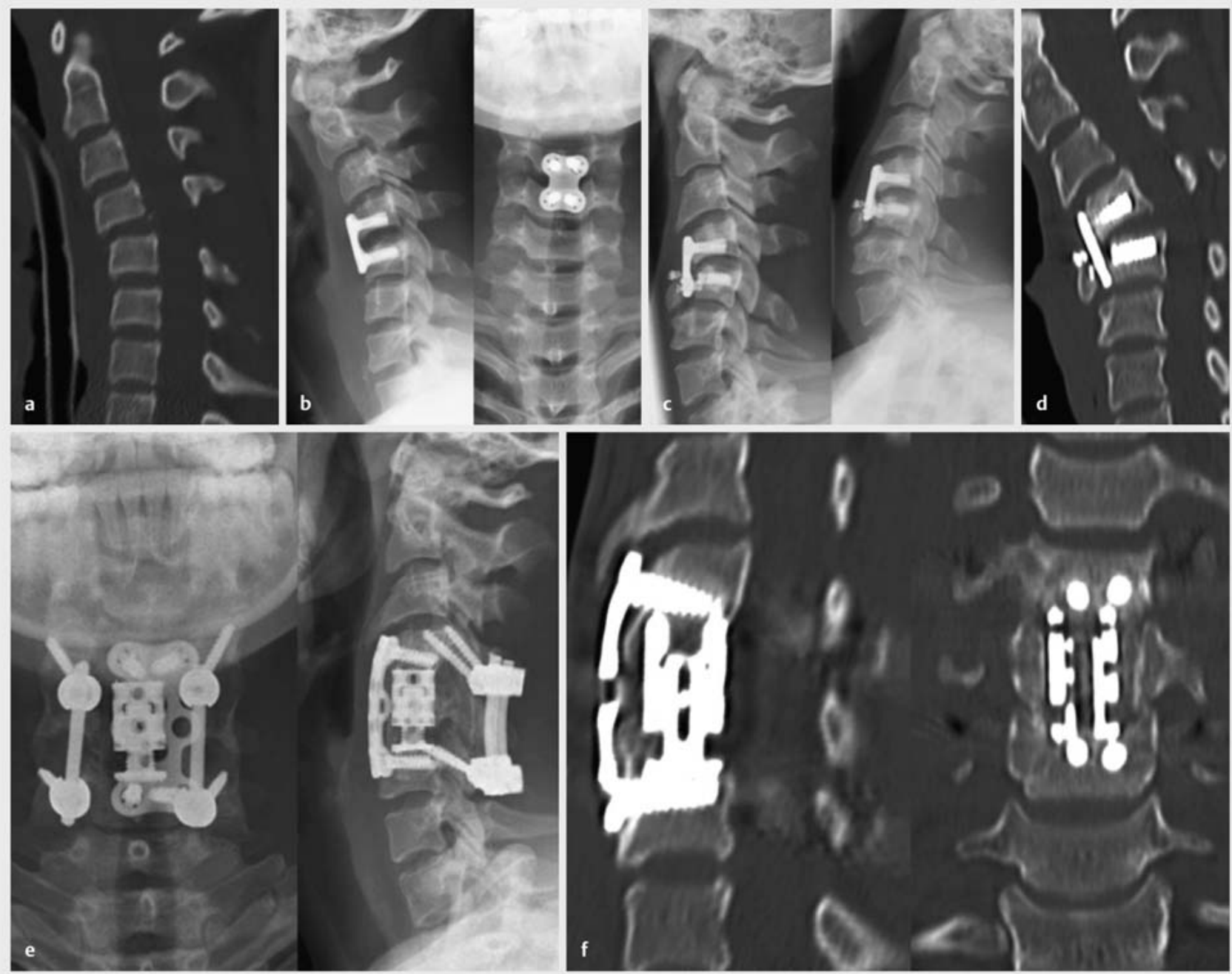


\section{Viszerale Läsionen}

Läsionen des Ösophagus oder des Pharynx/der Trachea sind selten und werden mit einer Inzidenz von bis zu 0,4\% bei hoher Mortalität von $20-50 \%$ in der Literatur beschrieben [11,16-18]. Diese können präparatorisch, durch den Druck des einliegenden Weichteilsperrers oder auch durch eine direkte Verletzung (z. B. mit der Fräse) entstehen. Nach entsprechender anatomischer Präparation sollte, wie zur Prävention von vaskulären Läsionen, der Weichteilsperrer unterhalb des M. Iongus colli platziert werden.

\section{Postoperative Komplikationen}

\section{Hämatom}

Ein postoperatives Hämatom stellt eine relevante Komplikation dar und wird in der Literatur mit einer Inzidenz von bis zu 5,6\% angegeben $[11,19]$. Durch die unmittelbare Nachbarschaft zu Ösophagus und Pharynx/Trachea kann sich eine lebensbedrohliche Verlegung des Atemweges entwickeln, was eine sofortige Entlastung des prävertebralen Hämatoms - ggf. noch vor Intubation - mit anschließender operativer Revision erfordert. Somit sollten die Patienten postoperativ streng hinsichtlich der Zeichen eines sich entwickelnden prävertebralen Hämatoms überwacht werden. Insbesondere werden ein zunehmender Halsumfang, Globusgefühl, zunehmende Schluckstörungen oder ein zunehmender inspiratorischer Stridor mit subjektiver Atemnot als dringliche Indikation für eine frühzeitige Revision - auch ohne vorherige Bildgebung gesehen. Ursächlich ist meist eine diffuse Blutung, daher wird bei der operativen Revision selten eine lokalisierte Blutung gefunden. Als wesentliche Prophylaxe einer postoperativen Nachblutung ist die sorgfältige intraoperative Blutstillung wie auch die Einlage einer prävertebralen Redon-Drainage zu empfehlen.

Im frühen postoperativen Verlauf ist eine neurologische Verschlechterung (z.B. Tetraparese/-plegie) durch ein sich entwickelndes epidurales Hämatom möglich. Auch hier ist eine sofortige Revision zu empfehlen. War der Patient direkt postoperativ neurologisch intakt, kann aus Gründen der Zeitersparnis auf eine vorherige Bildgebung verzichtet werden, da ein kompressiv wirkendes Epiduralhämatom hochwahrscheinlich ist.

\section{Infektion}

Infektiöse Wundheilungsstörungen nach ventraler Spondylodese sind selten. Bei Nachweis einer Wundinfektion sollte vor der Revision wie auch intraoperativ eine potenzielle viszerale Läsion (z. B. Ösophagusfistel) als Ursache der Infektion ausgeschlossen werden [20].

Cave

Cave: Eine übersehene Ösophagusperforation als Ursache einer Wundinfektion stellt unbehandelt eine potenziell lebensbedrohliche Komplikation dar.

\section{Implantatversagen/Pseudarthrose}

Die Pseudarthroserate kann insbesondere bei mehrsegmentaler Fusion durch die Verwendung von additiven Platten reduziert werden [21]. Ein Implantatversagen ist bei unzureichender Knochenqualität (z. B. Osteoporose), segmentale Instabilität bei Überdistraktion oder insuffizienter dorsaler Zuggurtung (AOSpine B2- oder C-Verletzung) möglich ( Abb. 14).

\section{Fazit}

Mittels ventraler Spondylodese können nahezu alle traumatischen Pathologien an der Halswirbelsäule versorgt werden. Ziel der Behandlung ist eine übungsstabile Versorgung, die in einer sicheren und dauerhaften Überbrückung des verletzten Bewegungssegmentes münden sollte. Nach adäquater Reposition und ventraler Diskektomie über einen weichteilschonenden ventralen Zugang kann dieses Ziel heutzutage durch die Kombination eines intervertebralen Platzhalters und zusätzlicher ventraler Plattenfixierung komplikationsarm erreicht werden.

Interessenkonflikt

Die Autoren erklären, dass sie innerhalb der vergangenen 3 Jahre in Beratungsgremien der Firmen Depuy Synthes (MS, FK), Siemens (FK), Vexim (MS) tätig waren und Vortragshonorare von den Firmen Depuy Synthes, Medtronic und Vexim erhalten haben.

\section{Autorinnen/Autoren:}

\section{Dr. med. Matti Scholz}

Zentrum für Wirbelsäulenchirurgie und Neurotraumatologie, BG Unfallklinik Frankfurt am Main

\section{Dr. med. Andreas Pingel}

Zentrum für Wirbelsäulenchirurgie und Neurotraumatologie, BG Unfallklinik Frankfurt am Main

\section{Dr. med. Philipp Schleicher}

Zentrum für Wirbelsäulenchirurgie und Neurotraumatologie, BG Unfallklinik Frankfurt am Main

Prof. Dr. med. Frank Kandziora

Zentrum für Wirbelsäulenchirurgie und Neurotraumatologie, BG Unfallklinik Frankfurt am Main

\section{Korrespondenzadresse}

\section{Dr. med. Matti Scholz}

Zentrum für Wirbelsäulenchirurgie und Neurotraumatologie BG-Unfallklinik Frankfurt am Main gGmbH

Friedberger Landstraße 430

60389 Frankfurt am Main

Tel.: 069/475-2020

Fax: 069/475-2018

matti.scholz@bgu-frankfurt.de 


\section{Literatur}

[1] Schleicher P, Scholz M, Kandziora F. et al. Therapieempfehlungen zur Versorgung von Verletzungen der subaxialen Halswirbelsäule. Z Orthop Unfall 2017; 155: 556-566. doi:10.1055/s0043-110855

[2] Scholz M, Schleicher P, Kandziora F. Verletzungen der subaxialen Halswirbelsäule. Orthopädie und Unfallchirurgie up2date 2015; 10: 429-450. doi:10.1055/s-0041-103862

[3] Herrmann HD. Metal plate fixation after anterior fusion of unstable fracture dislocations of the cervical spine. Acta Neurochir (Wien) 1975; 32: 101-111

[4] Bose B. Anterior cervical fusion using Caspar plating: analysis of results and review of the literature. Surg Neurol 1998; 49: 25-31

[5] Fehlings MG, Vaccaro A, Wilson JR et al. Early versus delayed decompression for traumatic cervical spinal cord injury: results of the Surgical Timing in Acute Spinal Cord Injury Study (STASCIS). PLoS One 2012; 7: e32037. doi:10.1371/journal. pone.0032037

[6] Panjabi MM, White AA 3rd. Basic biomechanics of the spine. Neurosurgery 1980; 7: 76-93

[7] Apfelbaum RI, Kriskovich MD, Haller JR. On the incidence, cause, and prevention of recurrent laryngeal nerve palsies during anterior cervical spine surgery. Spine 2000; 25: 29062912

[8] Scheufler KM, Diesing D. [Use of bone graft replacement in spinal fusions]. Orthopäde 2015; 44: 146-153. doi:10.1007/ s00132-014-3069-5

[9] Lehmann W, Blauth M, Briem D, el al. Biomechanical analysis of anterior cervical spine plate fixation systems with unicortical and bicortical screw purchase. Eur Spine J 2004; 13: 69-75. doi:10.1007/s00586-003-0578-z

[10] Pitzen T, Wilke HJ, Caspar W el al. Evaluation of a new monocortical screw for anterior cervical fusion and plating by a combined biomechanical and clinical study. Eur Spine J 1999; 8: 382-387. doi:10.1007/s005860050191

[11] Fountas KN, Kapsalaki EZ, Nikolakakos LG et al. Anterior cervical discectomy and fusion associated complications. Spine 2007; 32: 2310-2317. doi:10.1097/BRS.0b013e318154c57e
[12] Yasumoto Y, Abe Y, Tsutsumi S et al. [Rare complication of anterior spinal surgery: Horner syndrome]. No Shinkei Geka 2008; 36: 911-914

[13] Ebraheim NA, Lu J, Yang $\mathrm{H}$ et al. Vulnerability of the sympathetic trunk during the anterior approach to the lower cervical spine. Spine 2000; 25: 1603-1606

[14] Peng CW, Chou BT, Bendo JA et al. Vertebral artery injury in cervical spine surgery: anatomical considerations, management, and preventive measures. Spine | 2009; 9: 70-76. doi:10.1016/j.spinee.2008.03.006

[15] Burke JP, Gerszten PC, Welch WC. latrogenic vertebral artery injury during anterior cervical spine surgery. Spine J 2005; 5: 508-514. doi:10.1016/j.spinee.2004.11.015

[16] Pompili A, Canitano S, Caroli F et al. Asymptomatic esophageal perforation caused by late screw migration after anterior cervical plating: report of a case and review of relevant literature. Spine 2002; 27: E499-E502. doi:10.1097/01.BRS.00000353 09.04502 .52

[17] Vrouenraets BC, Been HD, Brouwer-Mladin R et al. Esophageal perforation associated with cervical spine surgery: report of two cases and review of the literature. Dig Surg 2004; 21: 246-249. doi:10.1159/000079495

[18] Korovessis P, Repantis T, Vitsas V et al. Cervical spondylodiscitis associated with oesophageal perforation: a rare complication after anterior cervical fusion. Eur J Orthop Surg Traumatol 2013; 23 (Suppl. 2): S159-S163. doi:10.1007/s00590-0121092-y

[19] Bertalanffy H, Eggert HR. Complications of anterior cervical discectomy without fusion in 450 consecutive patients. Acta Neurochir (Wien) 1989; 99: 41-50

[20] Christiano LD, Goldstein IM. Late prevertebral abscess after anterior cervical fusion. Spine 2011; 36: E798-E802. doi:10. 1097/BRS.0b013e3181fc9b09

[21] Fraser JF, Härtl R. Anterior approaches to fusion of the cervical spine: a metaanalysis of fusion rates. J Neurosurg Spine 2007; 6: 298-303. doi:10.3171/spi.2007.6.4.2

Bibliografie

DOI https://doi.org/10.1055/a-0582-7022

OP-JOURNAL 2018; 34: 138-150 @ Georg Thieme Verlag KG Stuttgart · New York ISSN 0178-1715 